\title{
Blinded Medication
}

National Cancer Institute

\section{Source}

National Cancer Institute. Blinded Medication. NCI Thesaurus. Code C142408.

Medicinal products, with or without the drug under study, which have the same appearance to ensure that neither the subject nor investigator can determine which medication is being administered. 